Research Article

\title{
Deterministic Epidemic Models for Ebola Infection with Time-Dependent Controls
}

\author{
Eric Okyere $\mathbb{D}^{D}$, Johnson De-Graft Ankamah (D), Anthony Kodzo Hunkpe, \\ and Dorcas Mensah \\ Department of Mathematics and Statistics, University of Energy and Natural Resources, Sunyani, Ghana \\ Correspondence should be addressed to Eric Okyere; eric.okyere@uenr.edu.gh
}

Received 27 March 2020; Accepted 16 June 2020; Published 4 July 2020

Academic Editor: Mustafa R. S. Kulenovic

Copyright ( $\odot 2020$ Eric Okyere et al. This is an open access article distributed under the Creative Commons Attribution License, which permits unrestricted use, distribution, and reproduction in any medium, provided the original work is properly cited.

\begin{abstract}
In this paper, we have studied epidemiological models for Ebola infection using nonlinear ordinary differential equations and optimal control theory. We considered optimal control analysis of SIR and SEIR models for the deadly Ebola infection using vaccination, treatment, and educational campaign as time-dependent control functions. We have applied indirect methods to study existing deterministic optimal control epidemic models for Ebola virus disease. These methods in optimal control are based on Hamiltonian function and Pontryagin's maximum principle to construct adjoint equations and optimality systems. The forward-backward sweep numerical scheme with the fourth-order Runge-Kutta method is used to solve the optimality system for the various control strategies. From our numerical illustrations, we can conclude that effective educational campaigns and vaccination of susceptible individuals as well as effective treatments of infected individuals can help reduce the disease transmission.
\end{abstract}

\section{Introduction}

The re-emergence of the Ebola virus disease in 2014 - 2016 in West Africa has been classified as the largest outbreak since the disease was first discovered in DRC in 1976 [1]. This highly infectious and deadly disease has claimed many lives and caused huge economic burden in the affected West Africa Countries. The recent 2018-2019 outbreak in eastern DRC is a very complex situation due to insecurities which is seriously affecting public health workers response activities [1].

Mathematical modeling of epidemics has contributed significantly in understanding the dynamical behaviour and control of infectious diseases [2]. The complex dynamics of Ebola virus disease has attracted the attention of many researchers who are interested in epidemiological modeling (see, e.g., [3-20]).

System of nonlinear equations that incorporates optimal control dynamics are key mathematical tools that are used in compartmental modeling to understand the spread of infectious diseases. Sharomi and Malik [21] conducted a comprehensive survey on optimal control modeling of several infectious diseases. Zakary et al. [22] formulated the epidemic model for controlling Ebola outbreak. A mathematical model for Ebola disease with time-dependent controls is proposed and numerically analyzed in [23]. Ahmad et al. [24] proposed and studied optimal control epidemic model for Ebola virus infection. Area et al. [25] introduced and studied the deterministic epidemic model for Ebola disease that incorporates optimal control dynamics with vaccination control. Grigorieva et al. [26] developed and analyzed a controlled dynamical model for Ebola virus infection.

Our present work is motivated by indirect methods in optimal control theory. These methods are based on Pontryagin's maximum principle and Hamiltonian function to construct adjoint equations and optimality systems for optimal control problems. Indirect methods have widely been applied by several authors, see the detailed survey on optimal modeling of infectious diseases by the authors in [21] and references therein. Recently, indirect methods in optimal control have also been used to study the dynamical behaviour and control of Zika virus disease (see, e.g., [27-30]). We are also inspired by the forward-backward 
numerical scheme with the fourth-order Runge-Kutta method for optimal control problems described in [31].

Rachah and Torres [32, 33] formulated and analyzed SIR and SEIR optimal control models for Ebola infection using direct methods. In both papers, they formulated objective functionals with dynamical state constrained equations. In [32], they proposed one optimal control strategy for the SIR model and the same authors in [33] proposed three different optimal control strategies for the SEIR model. After their models formulations, they applied the ACADO solver which is an automatic control and dynamic optimization tool to perform their numerical simulations. In our present work, we will apply indirect methods to study the mathematical models proposed in $[32,33]$. In this study, we will also propose one additional optimal control strategy for the SIR model. We will analytically construct Hamiltonian function and optimality system for the various optimal control strategies. We will then apply the forward-backward sweep method with the fourth-order Runge-Kutta method to perform numerical simulations in Matlab.

The rest of the paper is organized in three (3) more sections. In Section 2, we will introduce the classic SIR epidemic model to describe the dynamical behaviour of Ebola infection. We will then consider optimal control problems for the SIR model with two control strategies. In Section 3, we will present the basic SEIR epidemic model to describe the transmission dynamics of Ebola virus disease. We will further consider SEIR optimal control problems for this infectious disease with three different control strategies. In all these sections, we will formulate Hamiltonian functions and then apply Pontryagin's maximum principle to construct adjoint equations and optimality system for the various optimal control strategies. We will also perform numerical simulations for the optimality systems. Finally, we will present the conclusion in Section 4.

\section{SIR Model}

In this section, we introduce the classic SIR epidemic model to describe the dynamical behaviour of Ebola infection. The model assumes constant population size with no vital dynamics [2]. The population is divided into three different classes with $S(t), I(t)$, and $R(t)$ representing Susceptible, Infected, and Recovered individuals, respectively. As in [32], the nonlinear dynamical system describing Ebola infection is given by

$$
\begin{cases}\frac{\mathrm{d} S(t)}{\mathrm{d} t}=-\frac{\nu S(t) I(t)}{N}, & S(0)=S_{0} \geq 0 \\ \frac{\mathrm{d} I(t)}{\mathrm{d} t}=\frac{\nu S(t) I(t)}{N}-\delta I(t), & I(0)=I_{0} \geq 0 \\ \frac{\mathrm{d} R(t)}{\mathrm{d} t}=\delta I(t), & R(0)=R_{0} \geq 0\end{cases}
$$

with $S(t)+I(t)+R(t)=N$, where $v$ is the infection rate and $\delta$ is the recovery rate.
In this study, we will work with proportional quantities instead of the actual populations by scaling each state variable $(S, I, R)$ by the total population. For this purpose, we introduce new state variables $(s, i, r)$, which are expressed in terms of the original state variables and the total population given as follows:

$$
\left\{\begin{array}{l}
s(t)=\frac{S(t)}{N}, \\
i(t)=\frac{I(t)}{N}, \\
r(t)=\frac{R(t)}{N} .
\end{array}\right.
$$

Differentiating the scaling equation (2) with respect to time $t$ and using the unscaled model problem (1), we obtain the scaled SIR model describing the transmission dynamics of Ebola as follows:

$$
\begin{cases}\frac{\mathrm{d} s(t)}{\mathrm{d} t}=-v s(t) i(t), & s(0)=s_{0} \geq 0 \\ \frac{\mathrm{d} i(t)}{\mathrm{d} t}=v s(t) i(t)-\delta i(t), & i(0)=i_{0} \geq 0 \\ \frac{\mathrm{d} r(t)}{\mathrm{d} t}=\delta i(t), & r(0)=r_{0} \geq 0\end{cases}
$$

with $s(t)+i(t)+r(t)=1$, where the new state variables $s(t), i(t)$, and $r(t)$ represent proportions of Susceptible, Infected, and Recovered individuals, respectively.

As in [32], we will use this scaled SIR model (3) in Sections 2.1 and 2.2 to formulate optimal control models for Ebola infection with two different optimal control strategies.

2.1. SIR Model with Optimal Control Strategy 1. In this section, we consider a controlled dynamical system for the scaled SIR model (3), using vaccination as time-dependent control function, $\eta(t)$. For this optimal control problem, we want to reduce the number of infected individuals and the cost of vaccination. Therefore, in this control strategy, we minimize the objective functional $J(\eta)$ given by

$$
J(\eta)=\int_{0}^{t_{f}}\left[i(t)+\frac{B}{2} \eta^{2}(t)\right] \mathrm{d} t,
$$

subject to

$$
\begin{cases}\frac{\mathrm{d} s(t)}{\mathrm{d} t}=-v s(t) i(t)-\eta(t) s(t), & s(0)=s_{0} \geq 0, \\ \frac{\mathrm{d} i(t)}{\mathrm{d} t}=v s(t) s(t)-\delta i(t), & i(0)=i_{0} \geq 0, \\ \frac{\mathrm{d} r(t)}{\mathrm{d} t}=\delta i(t)+\eta(t) s(t), & r(0)=r_{0} \geq 0 .\end{cases}
$$

where the control set is given as follows: 
$\mathscr{G}_{1}=\left\{\eta: \eta(t)\right.$ is lebesgue measurable, $\left.0 \leq \eta(t) \leq 1, t \in\left[0, t_{f}\right]\right\}$,

and the positive parameter $B$ is the weight on cost of vaccination.
The Hamiltonian function corresponding to the objective functional (4) and the dynamic constrained state system (5) is given by

$$
H=i(t)+\frac{B}{2} \eta^{2}(t)+\varphi_{1}[-v s(t) i(t)-\eta(t) s(t)]+\varphi_{2}[\nu s(t) i(t)-\delta i(t)]+\varphi_{3}[\delta i(t)+\eta(t) s(t)] .
$$

We then apply the Maximum Principle proposed by authors in [34] to determine an optimal solution as follows.

Suppose that $(w, \eta)$ is an optimal solution for a controlled dynamical system, then there exist adjoint vector function $\varphi=\left(\varphi_{1}, \varphi_{2}, \ldots, \varphi_{n}\right)$, which satisfy the system below:

$$
\left\{\begin{array}{l}
\frac{\mathrm{d} w}{\mathrm{~d} t}=\frac{\partial H(t, w, \eta, \varphi)}{\partial \varphi}, \\
0=\frac{\partial H(t, w, \eta, \varphi)}{\partial \eta}, \\
\frac{\mathrm{d} \varphi}{\mathrm{d} t}=-\frac{\partial H(t, w, \eta, \varphi)}{\partial w} .
\end{array}\right.
$$

Following the constructed Hamiltonian function (7) and equation (8), we present the adjoint equations and the control characterisation as follows.

Theorem 1. Let $\eta^{*}$ be an optimal control and optimal state solutions $s^{*}, i^{*}$, and $r^{*}$ of the corresponding controlled $d y$ namical system (4)-(5) that minimize $J(\eta)$ over $\mathscr{G}_{1}$. Then, there exist adjoint variables $\varphi_{i}$ for $i=1,2,3$, which satisfy the system below:

$$
\left\{\begin{array}{l}
\frac{\mathrm{d} \varphi_{1}}{\mathrm{~d} t}=v i^{*}(t)\left(\varphi_{1}-\varphi_{2}\right)+\eta^{*}(t)\left(\varphi_{1}-\varphi_{3}\right) \\
\frac{\mathrm{d} \varphi_{2}}{\mathrm{~d} t}=-1+v s^{*}(t)\left(\varphi_{1}-\varphi_{2}\right)+\delta\left(\varphi_{2}-\varphi_{3}\right) \\
\frac{\mathrm{d} \varphi_{3}}{\mathrm{~d} t}=0
\end{array}\right.
$$

with transversality conditions $\varphi_{1}\left(t_{f}\right)=0, \varphi_{2}\left(t_{f}\right)=0$, and $\varphi_{3}\left(t_{f}\right)=0$, and the control $\eta^{*}$ satisfies the optimality condition:

$$
\eta^{*}(t)=\min \left\{\max \left\{0, \frac{s^{*}(t)}{B}\left(\varphi_{1}-\varphi_{3}\right)\right\}, 1\right\} .
$$

Proof. To derive the system of equations describing the adjoint variables and transversality conditions, we apply Pontryagin's Maximum Principle and the Hamiltonian function (7) as follows:

$$
\left\{\begin{array}{l}
\frac{\mathrm{d} \varphi_{1}}{\mathrm{~d} t}=-\frac{\partial H}{\partial s} \\
\frac{\mathrm{d} \varphi_{2}}{\mathrm{~d} t}=-\frac{\partial H}{\partial i} \\
\frac{\mathrm{d} \varphi_{3}}{\mathrm{~d} t}=-\frac{\partial H}{\partial r}
\end{array}\right.
$$

with

$$
\varphi_{i}\left(t_{f}\right)=0, \quad i=1,2,3 .
$$

By using the property of the control space $\mathscr{G}_{1}$ and solving the differential equation given by

$$
\frac{\partial H}{\partial \eta}=0,
$$

on the interior of the control set, the characterisation of the optimal control is obtained as given by equation (10).

2.2. SIR Model with Optimal Control Strategy 2. In this section, we present a controlled dynamical system by incorporating treatment control, $\eta_{1}(t)$, and educational campaign control, $\eta_{2}(t)$, into the scaled SIR model (3). For this optimal control problem, we want to reduce the number of infected individuals, the cost of educational campaign, and cost of treatment. Therefore, in this control strategy, we minimize the objective functional given as

$$
J\left(\eta_{1}, \eta_{2}\right)=\int_{0}^{t_{f}}\left[C_{1} i(t)+\frac{C_{2}}{2} \eta_{1}^{2}(t)+\frac{C_{3}}{2} \eta_{2}^{2}(t)\right] \mathrm{d} t
$$

which subjects to 


$$
\begin{cases}\frac{\mathrm{d} s(t)}{\mathrm{d} t}=-v s(t) i(t)-\eta_{2}(t) s(t), & s(0)=s_{0} \geq 0, \\ \frac{\mathrm{d} i(t)}{\mathrm{d} t}=v s(t) i(t)-\delta i(t)-\eta_{1}(t) i(t), & i(0)=i_{0} \geq 0, \\ \frac{\mathrm{d} r(t)}{\mathrm{d} t}=\delta i(t)+\eta_{1}(t) i(t)+\eta_{2}(t) s(t), & r(0)=r_{0} \geq 0,\end{cases}
$$

where the control set is given by

$$
\begin{array}{r}
\mathscr{G}_{2}=\left\{\left(\eta_{1}, \eta_{2}\right): \eta_{i}(t)\right. \text { is lebesgue measurable, } \\
\left.0 \leq \eta_{i}(t) \leq 1, t \in\left[0, t_{f}\right], \quad \text { for } i=1,2\right\},
\end{array}
$$

and $C_{1}$ is a positive constant to keep a balance in the size of $i(t)$. The positive parameters $C_{2}$ and $C_{3}$ are the weight on cost for treatments and educational campaigns.

The Hamiltonian function corresponding to equations (14) and (15) is given as

$$
\begin{aligned}
H= & C_{1} i(t)+\frac{C_{2}}{2} \eta_{1}^{2}(t)+\frac{C_{3}}{2} \eta_{2}^{2}(t)+\varphi_{1}\left[-\nu s(t) i(t)-\eta_{2}(t) s(t)\right] \\
& +\varphi_{2}\left[\nu s(t) i(t)-\delta i(t)-\eta_{1}(t) i(t)\right] \\
& +\varphi_{3}\left[\delta i(t)+\eta_{1}(t) i(t)+\eta_{2}(t) s(t)\right] .
\end{aligned}
$$

We then apply the Maximum Principle proposed by authors in [34] to determine an optimal solution.

Assume that $(w, \eta)$ is an optimal solution for a controlled dynamical system; then, there exist adjoint vector function $\varphi=\left(\varphi_{1}, \varphi_{2}, \ldots, \varphi_{n}\right)$, which satisfy the following equations:

$$
\left\{\begin{array}{l}
\frac{\mathrm{d} w}{\mathrm{~d} t}=\frac{\partial H(t, w, \eta, \varphi)}{\partial \varphi} \\
0=\frac{\partial H(t, w, \eta, \varphi)}{\partial \eta}, \\
\frac{\mathrm{d} \varphi}{\mathrm{d} t}=-\frac{\partial H(t, w, \eta, \varphi)}{\partial w}
\end{array}\right.
$$

Using the formulated Hamiltonian function (17) and equation (18), the adjoint equations and the control characterisation are presented in the following theorem.

Theorem 2. Let $\eta_{1}^{*}$ and $\eta_{2}^{*}$ be optimal control pair and optimal state solutions $s^{*}, i^{*}$, and $r^{*}$ of the corresponding controlled dynamical systems (14)-(15) that minimize $J\left(\eta_{1}, \eta_{2}\right)$ over $\mathscr{G}_{2}$. Then, there exist adjoint variables $\varphi_{i}$ for $i=1,2,3$, satisfying

$$
\left\{\begin{array}{l}
\frac{\mathrm{d} \varphi_{1}}{\mathrm{~d} t}=v i^{*}(t)\left(\varphi_{1}-\varphi_{2}\right)+\eta_{2}^{*}(t)\left(\varphi_{1}-\varphi_{3}\right) \\
\frac{\mathrm{d} \varphi_{2}}{\mathrm{~d} t}=-C_{1}+v s^{*}(t)\left(\varphi_{1}-\varphi_{2}\right)+\delta\left(\varphi_{2}-\varphi_{3}\right)+\eta_{1}^{*}(t)\left(\varphi_{2}-\varphi_{3}\right) \\
\frac{\mathrm{d} \varphi_{3}}{\mathrm{~d} t}=0
\end{array}\right.
$$

with transversality conditions $\varphi_{1}\left(t_{f}\right)=0, \varphi_{2}\left(t_{f}\right)=0$, and $\varphi_{3}\left(t_{f}\right)=0$ and the control pair $\eta_{1}^{*}$ and $\eta_{2}^{*}$ satisfies the optimality conditions:

$$
\left\{\begin{array}{l}
\eta_{1}^{*}(t)=\min \left\{\max \left\{0, \frac{i^{*}(t)}{C_{2}}\left(\varphi_{2}-\varphi_{3}\right)\right\}, 1\right\}, \\
\eta_{2}^{*}(t)=\min \left\{\max \left\{0, \frac{s^{*}(t)}{C_{3}}\left(\varphi_{1}-\varphi_{3}\right)\right\}, 1\right\} .
\end{array}\right.
$$

Remark 1. We remark that the proof of Theorem 2 and subsequent theorems in the next section of this study are similar to that of Theorem 1, and we therefore omit their proofs.

2.3. Numerical Simulations and Discussion. This section deals with numerical solutions of optimal control problems formulated in Sections 2.1 and 2.2. Analytical solution of the nonlinear system of differential equations with optimal control is a very hard task in mathematical modeling. Therefore, in this study, we apply the efficient forwardbackward sweep numerical scheme with the fourth-order Runge-Kutta method to solve the optimality systems formulated for the two optimal control strategies. This numerical scheme has extensively been described by the authors in [31], in their mathematical modeling textbook, which concerns with the applications of differential equations and optimal control theory. For our numerical simulations, we have adapted the same model parameter values $(\nu=0.2$ and $\delta=0.1)$ as in the work by the authors in [32] and initial conditions: $s_{0}=0.95, i_{0}=0.05$, and $r_{0}=0$. For the positive parameters in the objectives functionals, we have assumed that $B=5, C_{1}=1, C_{2}=5$, and $C_{3}=5$. In Figure 1 , it is clear that there is a significant decrease in the number of susceptible individuals with control strategies 1 and 2 than without control. Figure 2 shows solution paths for infected individuals with control strategies 1 and 2 and without control. This plot shows significant decrease in the infected individuals with control strategies 1 and 2 than without control. In Figure 3, there is a rapid increase in recovered individuals with control strategies 1 and 2 than without control. Figures 4 and 5 represent control functions for optimal control strategies 1 and 2, respectively. 


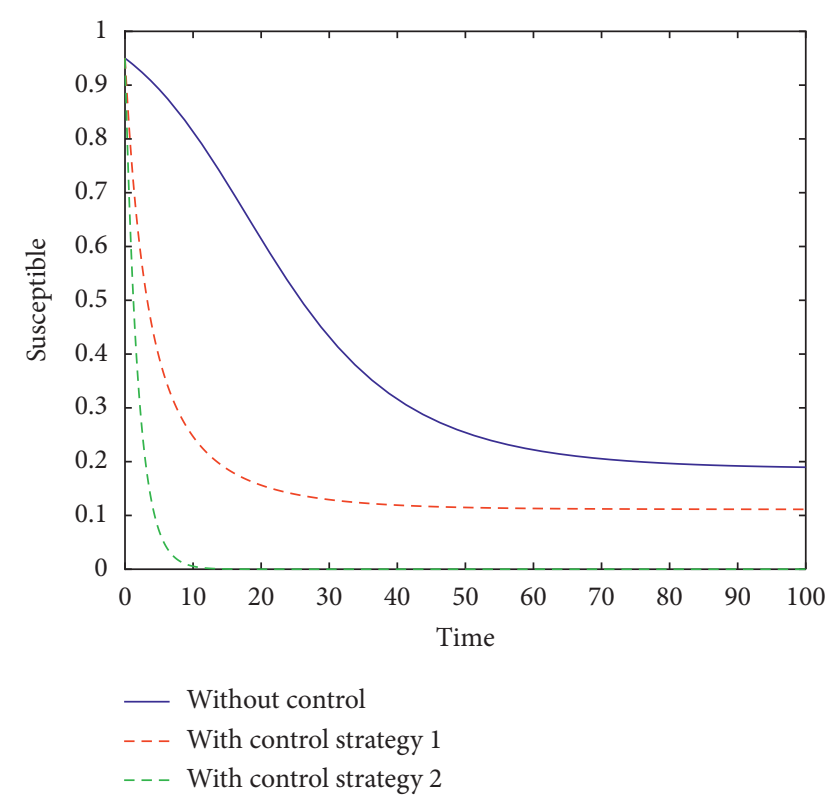

FIGURE 1: Solution paths for Susceptible individuals with two control strategies and without control, $v=0.2$ and $\delta=0.1$.

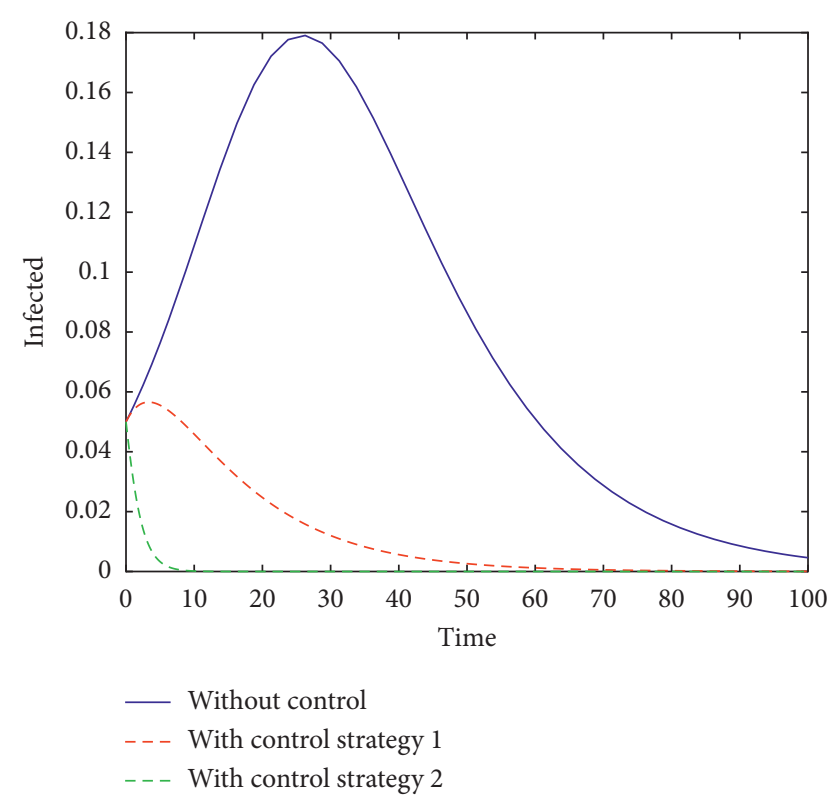

FIGURE 2: Solution paths for Infected individuals with two control strategies and without control, $v=0.2$ and $\delta=0.1$.

\section{SEIR Model}

In this section, we introduce the basic SEIR mathematical model to describe the transmission dynamics of Ebola infection. This model also assumes constant population size with no vital dynamics (birth and death rates). The total population is divided into four different classes with $S(t), E(t), E(t)$, and $R(t)$ representing Susceptible, Exposed, Infectious, and Recovered individuals, respectively. Following the compartmental modeling concepts in [2], the

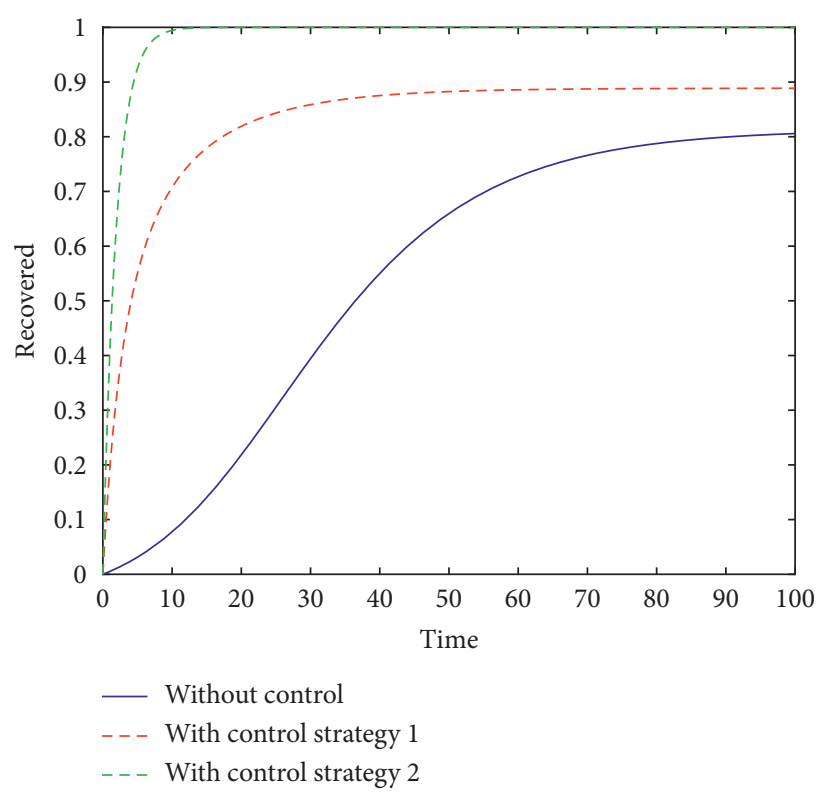

FIGURE 3: Solution paths for Recovered individuals with two control strategies and without control, $v=0.2$ and $\delta=0.1$.

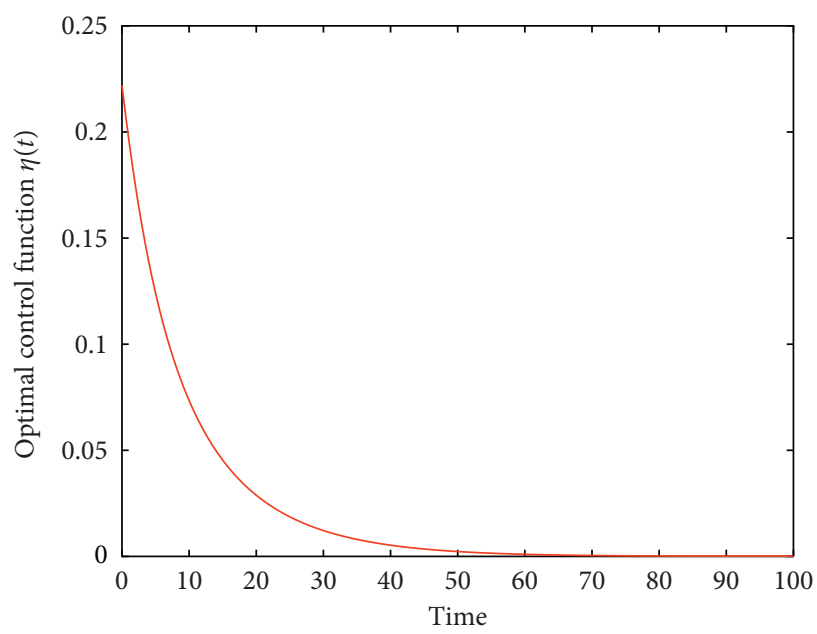

FIGURE 4: Optimal control function $\eta(t)$ for strategy 1.

nonlinear dynamical system describing Ebola virus disease is given by

$$
\begin{cases}\frac{\mathrm{d} S(t)}{\mathrm{d} t}=-\frac{\nu S(t) I(t)}{N}, & S(0)=S_{0} \geq 0 \\ \frac{\mathrm{d} E(t)}{\mathrm{d} t}=\frac{\nu S(t) I(t)}{N}-\rho E(t), & E(0)=E_{0} \geq 0 \\ \frac{\mathrm{d} I(t)}{\mathrm{d} t}=\rho E(t)-\delta I(t), & I(0)=I_{0} \geq 0 \\ \frac{\mathrm{d} R(t)}{\mathrm{d} t}=\delta I(t), & R(0)=R_{0} \geq 0\end{cases}
$$




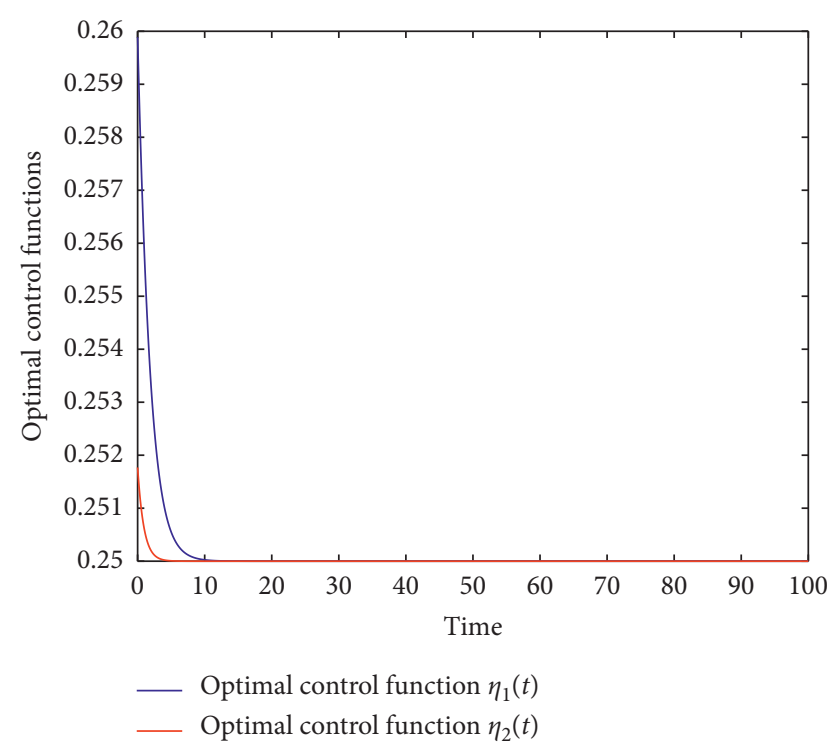

FIGURE 5: Optimal control functions $\eta_{1}(t)$ and $\eta_{2}(t)$ for strategy 2.

with $S(t)+E(t)+I(t)+R(t)=N$, where $v$ is the transmission rate, $\rho$ represent the infectious rate, and $\delta$ is the recovery rate.

As we carried out in Section 2, we will work with proportional quantities instead of the actual populations by scaling each state variable $(S(t), E(t), I(t), R(t))$ by the total population. We therefore introduce new state variables $(s(t), e(t), i(t), r(t))$, which are expressed in terms of the original state variables, and the total population is given by

$$
\left\{\begin{array}{l}
s(t)=\frac{S(t)}{N}, \\
e(t)=\frac{E(t)}{N}, \\
i(t)=\frac{I(t)}{N}, \\
r(t)=\frac{R(t)}{N} .
\end{array}\right.
$$

By differentiating the scaling equation (22) with respect to time $t$ and using the unscaled model problem (21), we obtain the scaled SEIR model as follows:

$$
\begin{cases}\frac{\mathrm{d} s(t)}{\mathrm{d} t}=-v \mathcal{S}(t) i(t), & s(0)=s_{0} \geq 0, \\ \frac{\mathrm{d} e(t)}{\mathrm{d} t}=v \mathcal{S}(t) i(t)-\rho e(t), & e(0)=e_{0} \geq 0, \\ \frac{\mathrm{d} i(t)}{\mathrm{d} t}=\rho e(t)-\delta i(t), & i(0)=i_{0} \geq 0 \\ \frac{\mathrm{d} r(r)}{\mathrm{d} t}=\delta i(t), & r(0)=r_{0} \geq 0\end{cases}
$$

with $s(t)+e(t)+i(t)+r(t)=1$, where the new state variables $s(t), e(t), i(t)$, and $r(t)$ represent proportions of Susceptible, Infected, and Recovered individuals, respectively.

As in [33], we will use this scaled SEIR model problem (23) in Sections 3.1, 3.2, and 3.3 to construct optimal control models for Ebola infection with three different control strategies.

3.1. SEIR Model with Optimal Control Strategy 1. In this section, we consider a controlled dynamical system for the scaled SEIR model (23), using vaccination as timedependent control function, $\eta(t)$. As we did in Section 2.1, our main objective for this optimal control problem is that we want to reduce the number of infected individuals and the cost of vaccination. Therefore, in this control strategy, we minimize the objective functional $J(\eta)$ given by

$$
J(\eta)=\int_{0}^{t_{f}}\left[i(t)+\frac{D}{2} \eta^{2}(t)\right] \mathrm{d} t,
$$

which subjects to

$$
\begin{cases}\frac{\mathrm{d} s(t)}{\mathrm{d} t}=-\nu s(t) i(t)-\eta(t) s(t), & s(0)=s_{0} \geq 0, \\ \frac{\mathrm{d} e(t)}{\mathrm{d} t}=\nu \mathcal{S}(t) i(t)-\rho e(t), & e(0)=e_{0} \geq 0, \\ \frac{\mathrm{d} i(t)}{\mathrm{d} t}=\rho e(t)-\delta i(t), & i(0)=i_{0} \geq 0 \\ \frac{\mathrm{d} r(t)}{\mathrm{d} t}=\delta i(t)+\eta(t) s(t), & r(0)=r_{0} \geq 0\end{cases}
$$

where the control set is given by

$$
\mathscr{G}_{3}=\left\{\eta: \eta(t) \text { is lebesgue measurable, } 0 \leq \eta(t) \leq 1, t \in\left[0, t_{f}\right]\right\} \text {, }
$$

and the positive constant $D$ is the weight on the cost of vaccination.

The Hamiltonian function $H$ is then given as follows:

$$
\begin{aligned}
H= & i(t)+\frac{D}{2} \eta^{2}(t)+\varphi_{1}[-\nu s(t) i(t)-\eta(t) s(t)] \\
& +\varphi_{2}[\nu s(t) i(t)-\rho e(t)] \\
& +\varphi_{3}[\rho e(t)-\delta i(t)]+\varphi_{4}[\delta i(t)+\eta(t) s(t)] .
\end{aligned}
$$

We then apply the Maximum Principle proposed by authors in [34] to determine an optimal solution.

Given that $(w, \eta)$ is an optimal solution for a controlled dynamical system, there exist adjoint vector function $\varphi=\left(\varphi_{1}, \varphi_{2}, \ldots, \varphi_{n}\right)$, which satisfy the following equations: 


$$
\left\{\begin{array}{l}
\frac{\mathrm{d} w}{\mathrm{~d} t}=\frac{\partial H(t, w, \eta, \varphi)}{\partial \varphi}, \\
0=\frac{\partial H(t, w, \eta, \varphi)}{\partial \eta}, \\
\frac{\mathrm{d} \varphi}{\mathrm{d} t}=-\frac{\partial H(t, w, \eta, \varphi)}{\partial w} .
\end{array}\right.
$$

Using the formulated Hamiltonian function (27) and equation (28), the adjoint equations and the control characterisation are presented in the following theorem.

Theorem 3. Let $\eta^{*}$ be an optimal control and optimal state solutions $s^{*}, e^{*}, i^{*}$, and $r^{*}$ of the corresponding controlled dynamical system (24)-(25) that minimize $J(\eta)$ over $\mathscr{G}_{3}$. Then, there exist adjoint variables $\varphi_{i}$ for $i=1,2,3,4$, satisfying

$$
\left\{\begin{array}{l}
\frac{\mathrm{d} \varphi_{1}}{\mathrm{~d} t}=v i^{*}(t)\left(\varphi_{1}-\varphi_{2}\right)+\eta^{*}(t)\left(\varphi_{1}-\varphi_{4}\right), \\
\frac{\mathrm{d} \varphi_{2}}{\mathrm{~d} t}=\rho\left(\varphi_{2}-\varphi_{3}\right), \\
\frac{\mathrm{d} \varphi_{3}}{\mathrm{~d} t}=-1+v s^{*}(t)\left(\varphi_{1}-\varphi_{2}\right)+\delta\left(\varphi_{3}-\varphi_{4}\right), \\
\frac{\mathrm{d} \varphi_{4}}{\mathrm{~d} t}=0,
\end{array}\right.
$$

with transversality conditions $\varphi_{1}\left(t_{f}\right)=0, \varphi_{2}\left(t_{f}\right)=0$, $\varphi_{3}\left(t_{f}\right)=0$, and $\varphi_{4}\left(t_{f}\right)=0$ and the control $\eta^{*}(t)$ satisfies the optimality condition:

$$
\eta^{*}(t)=\min \left\{\max \left\{0, \frac{s^{*}(t)}{D}\left(\varphi_{1}-\varphi_{4}\right)\right\}, 1\right\} .
$$

3.2. SEIR Model with Optimal Control Strategy 2. In this section, we present a controlled dynamical system for the scaled SEIR model (23), using vaccination as the time-dependent control function, $\eta(t)$. Our main objective for this strategy is to reduce the number of infected and exposed individuals as well as the cost of vaccination. Therefore, in this control strategy, we minimize the objective functional $J(\eta)$ given by

$$
J(\eta)=\int_{0}^{t_{f}}\left[K_{1} e(t)+K_{2} i(t)+\frac{K_{3}}{2} \eta^{2}(t)\right] \mathrm{d} t,
$$

which subjects to

$$
\begin{cases}\frac{\mathrm{d} s(t)}{\mathrm{d} t}=-v s(t) i(t)-\eta(t) s(t), & s(0)=s_{0} \geq 0, \\ \frac{\mathrm{d} e(t)}{\mathrm{d} t}=v s(t) i(t)-\rho e(t), & e(0)=e_{0} \geq 0, \\ \frac{\mathrm{d} i(t)}{\mathrm{d} t}=\rho e(t)-\delta i(t), & i(0)=i_{0} \geq 0, \\ \frac{\mathrm{d} r(t)}{\mathrm{d} t}=\delta i(t)+\eta(t) s(t), & r(0)=r_{0} \geq 0\end{cases}
$$

where the control set is given as follows:

$\mathscr{G}_{4}=\left\{\eta: \eta(t)\right.$ is lebesgue measurable, $\left.0 \leq \eta(t) \leq 1, t \in\left[0, t_{f}\right]\right\}$,

and the parameters $K_{1}$ and $K_{2}$ are positive constants associated with exposed individuals and infected individuals, respectively, and $K_{3}$ is a positive weight constant for control function $\eta(t)$.

The Hamiltonian $H$ is given by

$$
\begin{aligned}
H= & K_{1} e(t)+K_{2} i(t)+\frac{K_{3}}{2} \eta^{2}(t)+\varphi_{1}[-v s(t) i(t)-\eta(t) s(t)] \\
& +\varphi_{2}[\nu s(t) i(t)-\rho e(t)]+\varphi_{3}[\rho e(t)-\delta i(t)] \\
& +\varphi_{4}[\delta i+\eta(t) s(t)] .
\end{aligned}
$$

We then apply the Maximum Principle proposed by authors in [34] to determine an optimal solution.

Assume that $(w, \eta)$ is an optimal solution for a controlled dynamical system; then, there exist adjoint vector function $\varphi=\left(\varphi_{1}, \varphi_{2}, \ldots, \varphi_{n}\right)$, which satisfy the following equations:

$$
\left\{\begin{array}{l}
\frac{\mathrm{d} w}{\mathrm{~d} t}=\frac{\partial H(t, w, \eta, \varphi)}{\partial \varphi}, \\
0=\frac{\partial H(t, w, \eta, \varphi)}{\partial \eta}, \\
\frac{\mathrm{d} \varphi}{\mathrm{d} t}=-\frac{\partial H(t, w, \eta, \varphi)}{\partial w} .
\end{array}\right.
$$

Using the formulated Hamiltonian function (34) and equation (35), the adjoint equations and the control characterisation are given in the following theorem.

Theorem 4. Let $\eta^{*}$ be an optimal control and optimal state solutions $s^{*}, e^{*}, i^{*}$, and $r^{*}$ of the corresponding controlled dynamical system (31)-(32) that minimize $J(\eta)$ over $\mathscr{G}_{4}$. Then, there exist adjoint variables $\varphi_{i}$ for $i=1,2,3,4$, satisfying 


$$
\left\{\begin{array}{l}
\frac{\mathrm{d} \varphi_{1}}{\mathrm{~d} t}=v i^{*}(t)\left(\varphi_{1}-\varphi_{2}\right)+\eta^{*}(t)\left(\varphi_{1}-\varphi_{4}\right), \\
\frac{\mathrm{d} \varphi_{2}}{\mathrm{~d} t}=-K_{1}+\rho\left(\varphi_{2}-\varphi_{3}\right) \\
\frac{\mathrm{d} \varphi_{3}}{\mathrm{~d} t}=-K_{2}+v s^{*}(t)\left(\varphi_{1}-\varphi_{2}\right)+\delta\left(\varphi_{3}-\varphi_{4}\right), \\
\frac{\mathrm{d} \varphi_{4}}{\mathrm{~d} t}=0
\end{array}\right.
$$

with transversality conditions $\varphi_{1}\left(t_{f}\right)=0, \varphi_{2}\left(t_{f}\right)=0$, $\varphi_{3}\left(t_{f}\right)=0$, and $\varphi_{4}\left(t_{f}\right)=0$ and the control function $\eta^{*}$ is given by

$$
\eta^{*}(t)=\min \left\{\max \left\{0, \frac{s^{*}(t)}{K_{3}}\left(\varphi_{1}-\varphi_{4}\right)\right\}, 1\right\} .
$$

3.3. SEIR Model with Optimal Control Strategy 3. In this section, we present a controlled dynamical problem by incorporating treatment control, $\eta_{1}(t)$, and educational campaign control, $\eta_{2}(t)$, into the SEIR model (23). As we presented in Section 2.2, our main aim for this controlled problem is to minimize the number of infected individuals and the cost of educational campaigns and treatment. Therefore, in this control strategy, we minimize the objective functional given by

$$
J\left(\eta_{1}, \eta_{2}\right)=\int_{0}^{t_{f}}\left[D_{1} i(t)+\frac{D_{2}}{2} \eta_{1}^{2}(t)+\frac{D_{3}}{2} \eta_{2}^{2}(t)\right] \mathrm{d} t,
$$

which subjects to

$$
\begin{cases}\frac{\mathrm{d} s(t)}{\mathrm{d} t}=-v s(t) i(t)-\eta_{2}(t) s(t), & s(0)=s_{0} \geq 0, \\ \frac{\mathrm{de}(t)}{\mathrm{d} t}=v s(t) i(t)-\rho e(t), & e(0)=e_{0} \geq 0, \\ \frac{\mathrm{d} i(t)}{\mathrm{d} t}=\rho e(t)-\delta i(t)-\eta_{1}(t) i(t), & i(0)=i_{0} \geq 0, \\ \frac{\mathrm{d} r(t)}{\mathrm{d} t}=\delta i(t)+\eta_{1}(t) i(t)+\eta_{2}(t) s(t), & r(0)=r_{0} \geq 0,\end{cases}
$$

where the control set is given as follows:

$$
\mathscr{G}_{5}=\left\{\left(\eta_{1}, \eta_{2}\right): \eta_{i}(t) \text { is lebesgue measurable, } 0 \leq \eta_{i}(t) \leq 1, t \in\left[0, t_{f}\right] \text { for } i=1,2\right\} \text {, }
$$

where $D_{1}$ is the weight constant on infected individuals and $D_{2}$ and $D_{3}$ are also positive weight parameters associated with controls $\eta_{1}(t)$ and $\eta_{2}(t)$, respectively.

The Hamiltonian function $H$ is given as

$$
\begin{aligned}
H= & \left.D_{1} i(t)\right]+\frac{D_{2}}{2} \eta_{1}^{2}(t)+\frac{D_{3}}{2} \eta_{2}^{2}(t)+\varphi_{1}\left[-v s(t) i(t)-\eta_{2}(t) s(t)\right] \\
& +\varphi_{2}[\nu s(t) i(t)-\rho e(t)]+\varphi_{3}\left[\rho e(t)-\delta i(t)-\eta_{1}(t) i(t)\right] \\
& +\varphi_{4}\left[\delta i(t)+\eta_{1}(t) i(t)+\eta_{2}(t) s(t)\right] .
\end{aligned}
$$

We then apply the Maximum Principle proposed by authors in [34] to determine an optimal solution.

Assume that $(w, \eta)$ is an optimal solution for a controlled dynamical system; then, there exist adjoint vector function $\varphi=\left(\varphi_{1}, \varphi_{2}, \ldots, \varphi_{n}\right)$, which satisfy the following equations:

$$
\left\{\begin{array}{l}
\frac{\mathrm{d} w}{\mathrm{~d} t}=\frac{\partial H(t, w, \eta, \varphi)}{\partial \varphi} \\
0=\frac{\partial H(t, w, \eta, \varphi)}{\partial \eta}, \\
\frac{\mathrm{d} \varphi}{\mathrm{d} t}=-\frac{\partial H(t, w, \eta, \varphi)}{\partial w}
\end{array}\right.
$$

Using the formulated Hamiltonian function (41) and equation (42), the adjoint equations and the control characterisation are presented in the following theorem.

Theorem 5. Let $\eta_{1}^{*}$ and $\eta_{2}^{*}$ be optimal control pair and optimal state solutions $s^{*}, e^{*}, i^{*}$, and $r^{*}$ of the corresponding controlled dynamical system (38)-(39) that minimize $J\left(\eta_{1}, \eta_{2}\right)$ over $\mathscr{G}_{5}$. Then, there exist adjoint variables $\varphi_{i}$ for $i=1,2,3,4$, satisfying

$$
\left\{\begin{array}{l}
\frac{\mathrm{d} \varphi_{1}}{\mathrm{~d} t}=v i^{*}(t)\left(\varphi_{1}-\varphi_{2}\right)+\eta_{2}^{*}(t)\left(\varphi_{1}-\varphi_{4}\right), \\
\frac{\mathrm{d} \varphi_{2}}{\mathrm{~d} t}=\rho\left(\varphi_{2}-\varphi_{3}\right), \\
\frac{\mathrm{d} \varphi_{3}}{\mathrm{~d} t}=-D_{1}+v \mathrm{~s}^{*}(t)\left(\varphi_{1}-\varphi_{2}\right)+\delta\left(\varphi_{3}-\varphi_{4}\right)+\eta_{1}^{*}(t)\left(\varphi_{3}-\varphi_{4}\right), \\
\frac{\mathrm{d} \varphi_{4}}{\mathrm{~d} t}=0,
\end{array}\right.
$$

with transversality conditions $\varphi_{1}\left(t_{f}\right)=0, \varphi_{2}\left(t_{f}\right)=0$, $\varphi_{3}\left(t_{f}\right)=0$, and $\varphi_{4}\left(t_{f}\right)=0$, and the control pair $\eta_{1}^{*}$ and $\eta_{2}^{*}$ satisfies the optimality condition: 


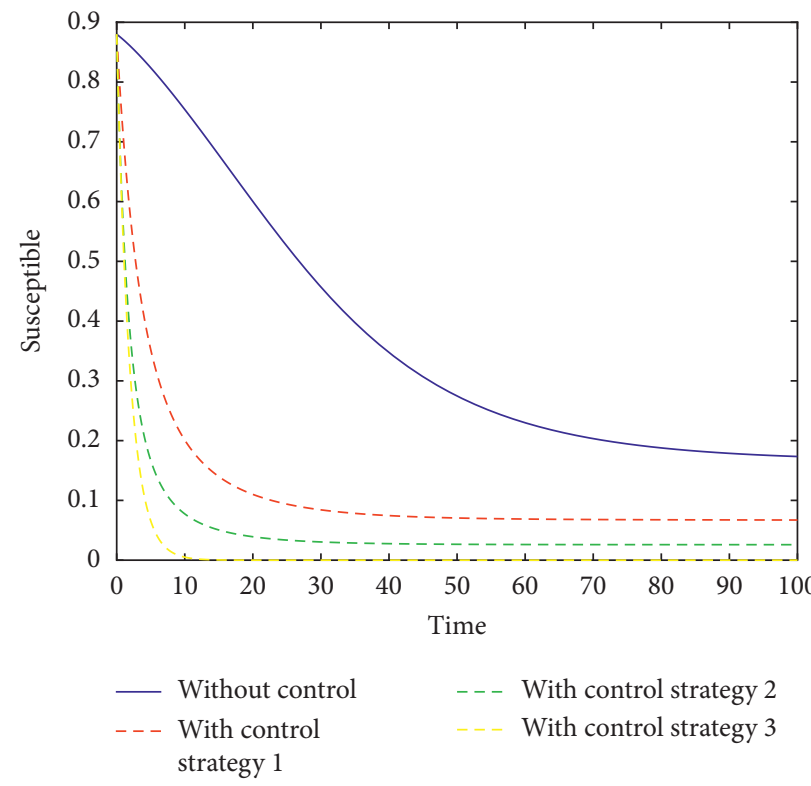

FIgURE 6: Solution path for Susceptible individuals with three control strategies and without control, $v=0.2, \rho=0.1887$, and $\delta=0.1$.

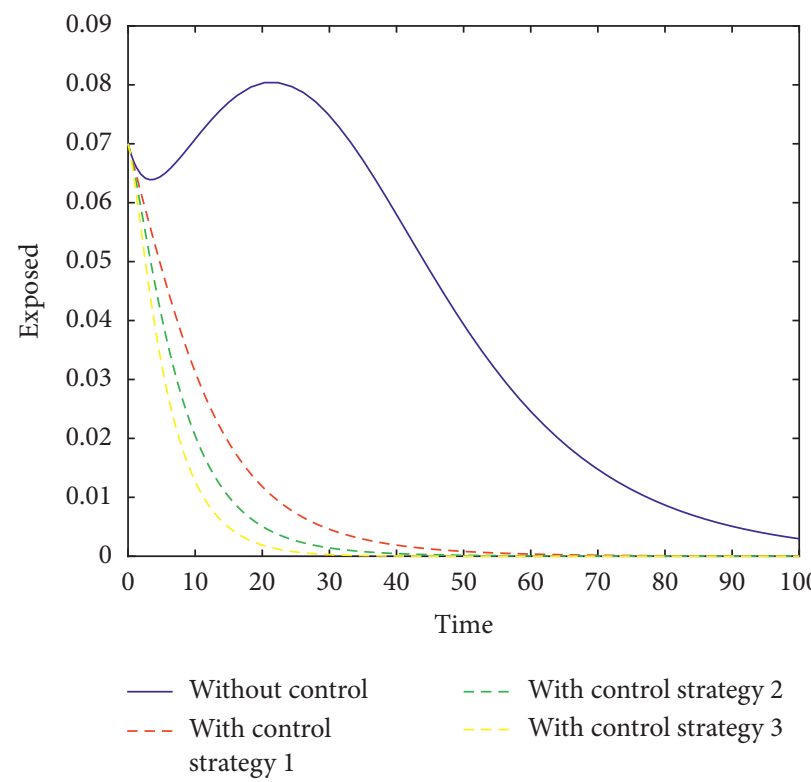

FIGURE 7: Solution paths for Exposed individuals with three control strategies and without control, $\nu=0.2, \rho=0.1887$, and $\delta=0.1$.

$$
\left\{\begin{array}{l}
\eta_{1}^{*}(t)=\min \left\{\max \left\{0, \frac{i^{*}(t)}{D_{2}}\left(\varphi_{3}-\varphi_{4}\right)\right\}, 1\right\}, \\
\eta_{2}^{*}(t)=\min \left\{\max \left\{0, \frac{s^{*}(t)}{D_{3}}\left(\varphi_{1}-\varphi_{4}\right)\right\}, 1\right\} .
\end{array}\right.
$$

3.4. Numerical Simulations and Discussion. As we did in Section 2.3, we have used the forward-backward sweep numerical scheme with the fourth-order Runge-Kutta method to solve the optimality systems formulated for the three optimal control strategies. For our numerical simulations, we have adapted the same model parameter values $(\nu=0.2, \delta=0.1$, and $\rho=0.1887)$ as in the work by the authors in [33] with initial conditions: $s_{0}=0.88, e_{0}=0.07, i_{0}=0.05$, and $r_{0}=0$. We have assumed $D=5, K_{1}=1, K_{2}=5, K_{3}=5, D_{1}=1, D_{2}=5$, and $D_{3}=5$. Figure 6 shows solution paths for susceptible individuals with control strategies 1,2 , and 3 and without control. It is clear from the plot that there is a significant decrease in the number of susceptible individuals with control strategies than without control. In Figure 7, there is a rapid decrease in the number of exposed individuals with 


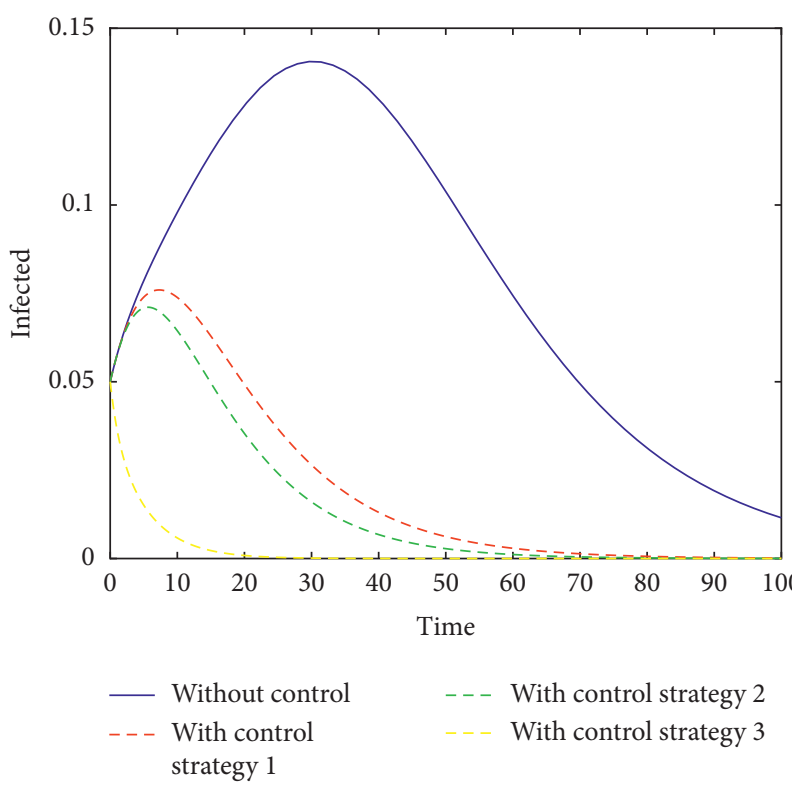

FIGURE 8: Solution paths for Infected individuals with three control strategies and without control, $\nu=0.2, \rho=0.1887$, and $\delta=0.1$.

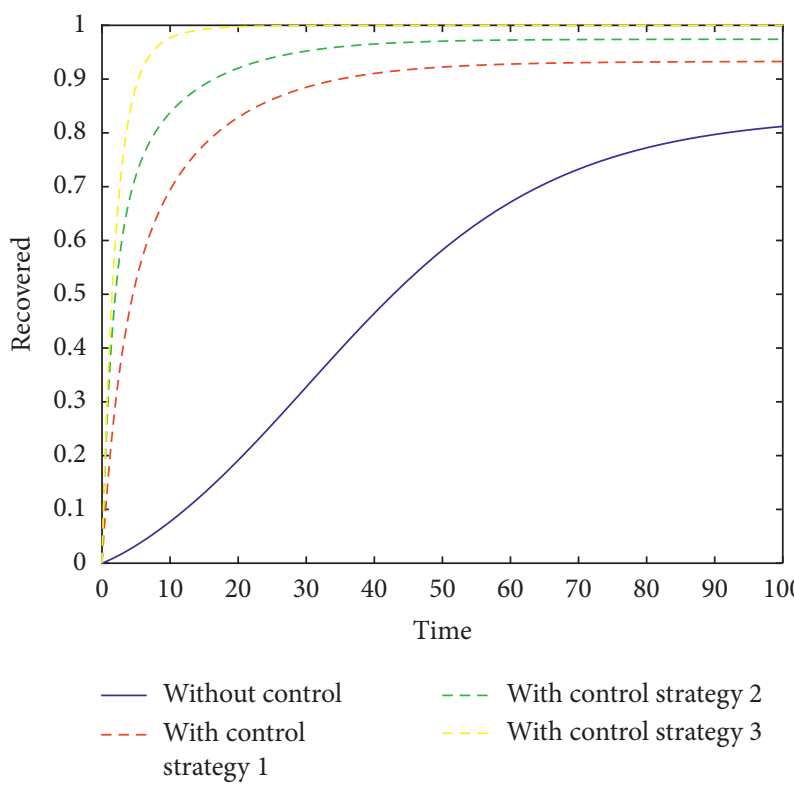

FIGURE 9: Solution paths for Recovered individuals with three control strategies and without control, $v=0.2, \rho=0.1887$, and $\delta=0.1$.

control strategies 1, 2, and 3 than without control. A similar effect can be observed in Figure 8 for the infected individuals. In Figure 9, there is a rapid increase in recovered individuals with control strategies 1,2 , and 3 than without control. Figures 10-12 represent optimal control functions for strategies 1, 2, and 3, respectively.

\section{Conclusion}

In this paper, we have studied epidemiological models for Ebola virus disease using nonlinear system of ordinary

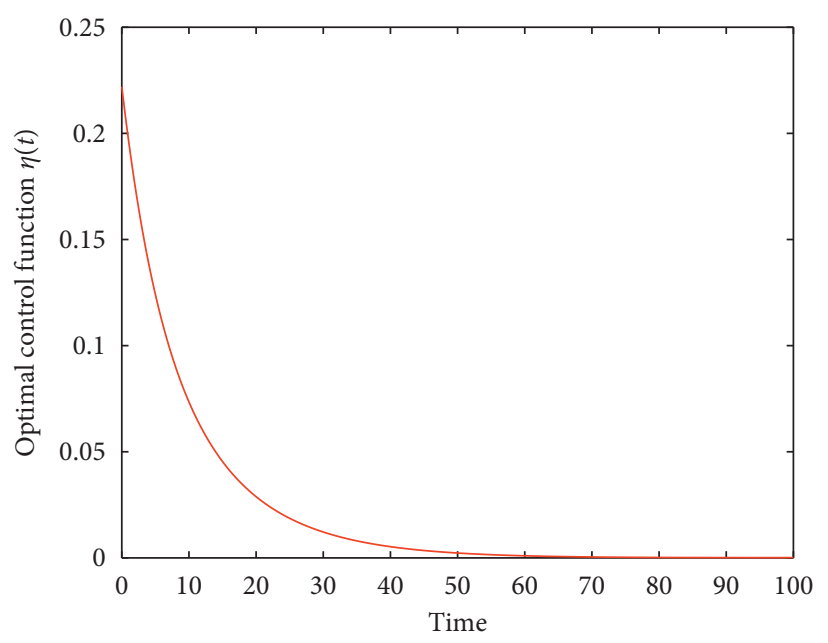

Figure 10: Optimal control function $\eta(t)$ for strategy 1 .

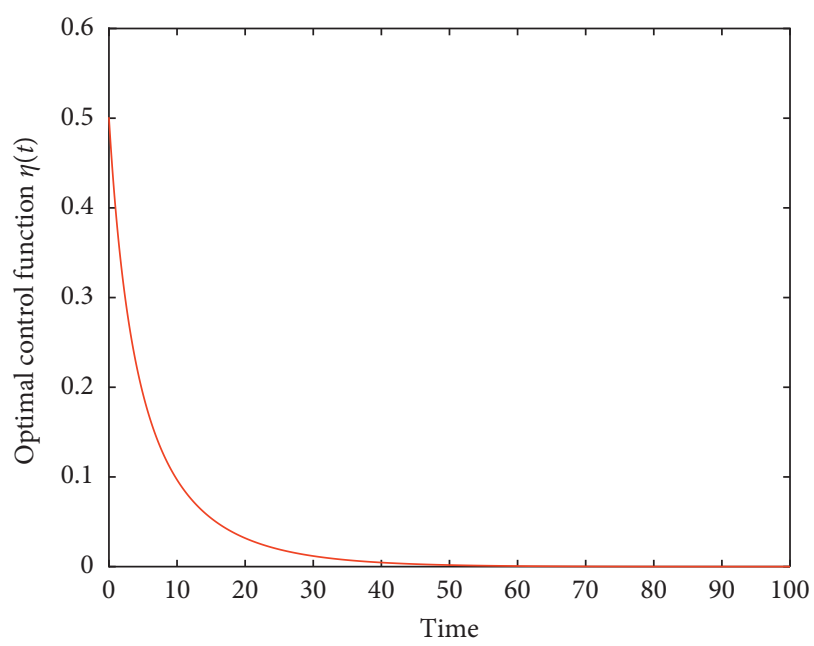

Figure 11: Optimal control functions $\eta(t)$ for strategy 2.

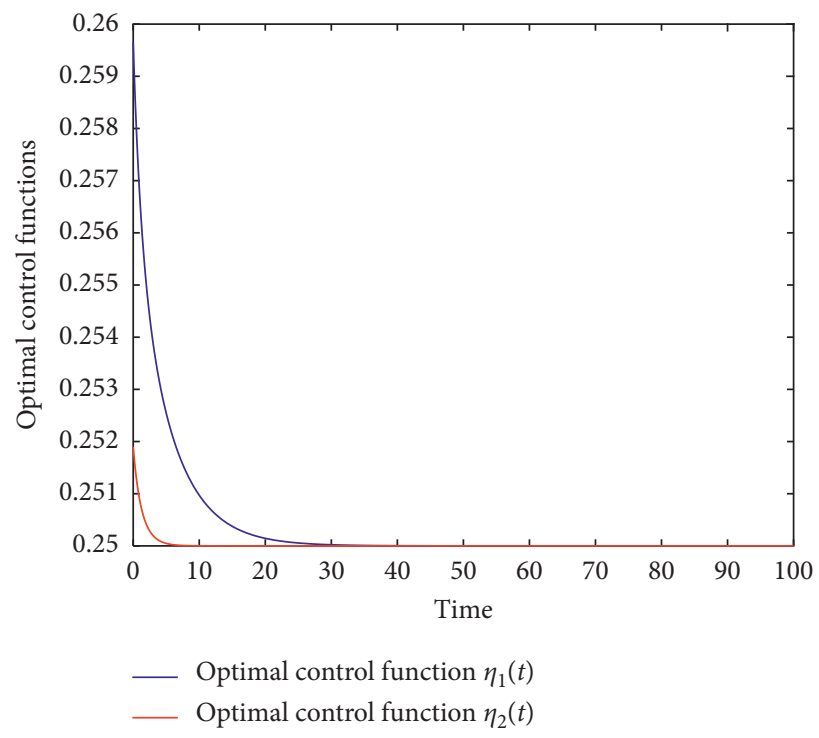

FIGURE 12: Optimal control functions $\eta_{1}(t)$ and $\eta_{2}(t)$ for strategy 3 . 
differential equation and optimal control theory. We have used indirect methods in optimal control to study existing mathematical models proposed by the authors in [32, 33]. Using Pontryagin's maximum principle and Hamiltonian function, we have derived adjoint equations and optimality system for the various optimal control strategies. From the simulation results, we observed that the SIR model with optimal control strategies shows significant decrease in the proportions of infected and susceptible individuals and a rapid increase in the recovered individuals compared to the SIR model without control. A similar effect was observed in the SEIR model with control strategies where there was a significant decrease in Susceptible, Exposed, and Infected individuals and a rapid increase in the Recovered individuals. Our numerical results are similar to the once generated by the authors in $[32,33]$ who applied direct methods in optimal control for their mathematical models. Therefore, following the numerical results, we can conclude that effective educational campaigns and vaccination of susceptible individuals as well as effective treatments of infected individuals can help reduce the disease transmission. In this work, we have also analytically and numerically studied one additional optimal control strategy for the SIR epidemic model.

\section{Data Availability}

Our mathematical modeling does not include data. All parameter values that were used for our simulations have been cited accordingly.

\section{Conflicts of Interest}

The authors declare that there are no conflicts of interest regarding the publication of this paper.

\section{References}

[1] WHO, Ebola Virus Disease, WHO, Geneva, Switzerland, 2019, https://www.who.int/news-room/fact-sheets/detail/ebolavirus-disease.

[2] H. W. Hethcote, "The mathematics of infectious diseases," SIAM Review, vol. 42, no. 4, pp. 599-653, 2000.

[3] T. W. Tulu, B. Tian, and Z. Wu, "Mathematical modeling, analysis and Markov chain Monte Carlo simulation of ebola epidemics," Results in Physics, vol. 7, pp. 962-968, 2017.

[4] F. Dong, D. Xu, Z. Wang, and M. Dong, "Evaluation of ebola spreading in west africa and decision of optimal medicine delivery strategies based on mathematical models, Infection, Genetics and Evolution, vol. 36, pp. 35-40, 2015.

[5] C. L. Althaus, "Estimating the reproduction number of ebola virus (ebov) during the 2014 outbreak in West Africa," PLoS Currents, vol. 6, 2014.

[6] G. Chowell and H. Nishiura, "Transmission dynamics and control of ebola virus disease (evd): a review," BMC Medicine, vol. 12, no. 1, p. 196, 2014.

[7] G. A. Ngwa and M. I. Teboh-Ewungkem, "A mathematical model with quarantine states for the dynamics of ebola virus disease in human populations," Computational and Mathematical Methods in Medicine, vol. 2016, Article ID 9352725, 29 pages, 2016.
[8] J. S. Weitz and J. Dushoff, "Modeling post-death transmission of ebola: challenges for inference and opportunities for control," Scientific Reports, vol. 5, no. 1, p. 8751, 2015.

[9] D. Ndanguza, I. S. Mbalawata, H. Haario, and J. M. Tchuenche, "Analysis of bias in an ebola epidemic model by extended kalman filter approach," Mathematics and Computers in Simulation, vol. 142, pp. 113-129, 2017.

[10] P. Diaz, P. Constantine, K. Kalmbach, E. Jones, and S. Pankavich, "A modified SEIR model for the spread of Ebola in Western Africa and metrics for resource allocation," Applied Mathematics and Computation, vol. 324, pp. 141-155, 2018.

[11] A. Atangana and E. F. D. Goufo, "On the mathematical analysis of ebola hemorrhagic fever: deathly infection disease in west african countries," BioMed Research International, vol. 2014, Article ID 261383, 7 pages, 2014.

[12] S. Jiang, K. Wang, C. Li et al., "Mathematical models for devising the optimal ebola virus disease eradication," Journal of Translational Medicine, vol. 15, no. 1, p. 124, 2017.

[13] T. Berge, J. M.-S. Lubuma, G. M. Moremedi, N. Morris, and R. Kondera-Shava, "A simple mathematical model for ebola in africa," Journal of Biological Dynamics, vol. 11, no. 1, pp. 42-74, 2017.

[14] Z.-Q. Xia, S.-F. Wang, S.-L. Li et al., "Modeling the transmission dynamics of ebola virus disease in Liberia," Scientific Reports, vol. 5, no. 1, 2015.

[15] A. Khan, M. Naveed, M. Dur-e-Ahmad, and M. Imran, "Estimating the basic reproductive ratio for the Ebola outbreak in Liberia and Sierra Leone," Infectious Diseases of Poverty, vol. 4, no. 1, p. 13, 2015.

[16] G. Chowell, N. W. Hengartner, C. Castillo-Chavez, P. W. Fenimore, and J. M. Hyman, "The basic reproductive number of Ebola and the effects of public health measures: the cases of Congo and Uganda," Journal of Theoretical Biology, vol. 229, no. 1, pp. 119-126, 2004.

[17] E. F. D. Goufo, M. K. Pene, and S. Mugisha, "Stability analysis of epidemic models of ebola hemorrhagic fever with nonlinear transmission," Journal of Nonlinear Sciences \& Applications (JNSA), vol. 9, no. 6, pp. 4191-4205, 2016.

[18] F. B. Agusto, M. I. Teboh-Ewungkem, and A. B. Gumel, "Mathematical assessment of the effect of traditional beliefs and customs on the transmission dynamics of the 2014 ebola outbreaks," BMC Medicine, vol. 13, no. 1, p. 96, 2015.

[19] D. Luo, R. Zheng, D. Wang et al., "Effect of sexual transmission on the west africa ebola outbreak in 2014: a mathematical modelling study," Scientific Reports, vol. 9, no. 1, 2019.

[20] A. Dénes and A. B. Gumel, "Modeling the impact of quarantine during an outbreak of ebola virus disease," Infectious Disease Modelling, vol. 4, pp. 12-27, 2019.

[21] O. Sharomi and T. Malik, "Optimal control in epidemiology," Annals of Operations Research, vol. 251, no. 1-2, pp. 55-71, 2017.

[22] O. Zakary, M. Rachik, and I. Elmouki, "A multi-regional epidemic model for controlling the spread of ebola: awareness, treatment, and travel-blocking optimal control approaches," Mathematical Methods in the Applied Sciences, vol. 40, no. 4, pp. 1265-1279, 2017.

[23] E. Bonyah, K. Badu, and S. K. Asiedu-Addo, "Optimal control application to an ebola model," Asian Pacific Journal of Tropical Biomedicine, vol. 6, no. 4, pp. 283-289, 2016.

[24] M. D. Ahmad, M. Usman, A. Khan, and M. Imran, "Optimal control analysis of ebola disease with control strategies of 
quarantine and vaccination," Infectious Diseases of Poverty, vol. 5 , no. 1, p. 72, 2016.

[25] I. Area, F. Ndaïrou, F. NdaÏrou, J. J. Nieto, and C. J. Silva, "Ebola model and optimal control with vaccination constraints," Journal of Industrial \& Management Optimization, vol. 14, no. 2, pp. 427-446, 2018.

[26] E. V. Grigorieva, P. B. Deignan, and E. N. Khailov, "Optimal control problem for a seir type model of ebola epidemics," Revista de Matemática: Teoría y Aplicaciones, vol. 24, no. 1, pp. 79-96, 2017.

[27] E. Bonyah, M. A. Khan, K. O. Okosun, and S. Islam, "A theoretical model for zika virus transmission," PLoS One, vol. 12, no. 10, Article ID e0185540, 2017.

[28] M. A. Khan, S. W. Shah, S. Ullah, and J. F. Gómez-Aguilar, "A dynamical model of asymptomatic carrier zika virus with optimal control strategies," Nonlinear Analysis: Real World Applications, vol. 50, pp. 144-170, 2019.

[29] T. Y. Miyaoka, S. Lenhart, and J. C. A. Meyer, "Optimal control of vaccination ina vector-borne reaction-diffusion model applied to zika virus," Journal of Mathematical Biology, vol. 79, no. 3, pp. 1077-1104, 2019.

[30] E. Bonyah, M. A. Khan, K. O. Okosun, and J. F. GómezAguilar, "On the co-infection of dengue fever and Zika virus," Optimal Control Applications and Methods, vol. 40, no. 3, pp. 394-421, 2019.

[31] S. Lenhart and J. T. Workman, Optimal Control Applied to Biological Models, Chapman and Hall/CRC, Boca Raton, FL, USA, 2007.

[32] A. Rachah and D. F. M. Torres, "Mathematical modelling, simulation, and optimal control of the 2014 ebola outbreak in West Africa," Discrete Dynamics in Nature and Society, vol. 2015, Article ID 842792, 9 pages, 2015.

[33] A. Rachah and D. F. M. Torres, "Dynamics and optimal control of ebola transmission," Mathematics in Computer Science, vol. 10, no. 3, pp. 331-342, 2016.

[34] L. S. Pontryagin, V. G. Boltyanskii, R. V. Gamkrelidze, and E. F. Mishchenko, The Mathematical Theory of Optimal Processes, John Wiley \& Sons, New York, NY, USA, 1962. 